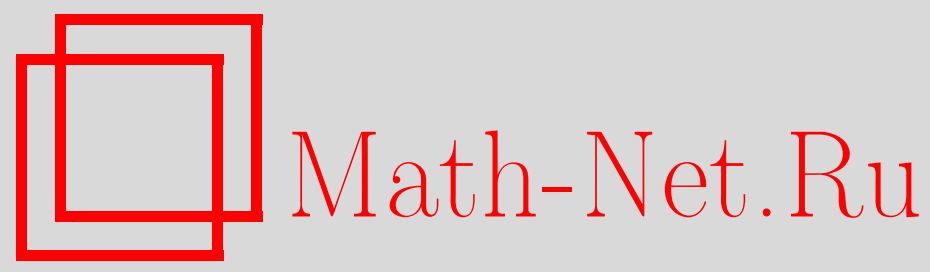

В. П. Громов, О полноте системы значений голоморфной вектор-функции в пространстве Фреше, Матем. заметки, 2003, том 73, выпуск 6, 827-840

DOI: https://doi.org/10.4213/mzm230

Использование Общероссийского математического портала Math-Net.Ru подразумевает, что вы прочитали и согласны с пользовательским соглашением http://www.mathnet.ru/rus/agreement

Параметры загрузки:

IP : 54.157 .27 .8

26 апреля 2023 г., 15:01:38

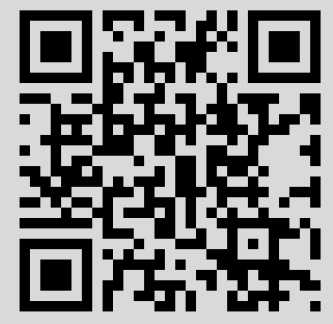




\title{
О ПОЛНОТЕ СИСТЕМЫ ЗНАЧЕНИЙ ГОЛОМОРФНОЙ ВЕКТОР-ФУНКЦИИ В ПРОСТРАНСТВЕ ФРЕШЕ
}

\author{
В.П. Громов
}

Установлены необходимые и достаточные условия полноты системы значений голоморфной вектор-функции в пространстве Фреше. Полученные результаты иллюстрируются разнообразньми примерами.

Библиография: 21 название.

Введение. Проблемы аппроксимации посредством функций, отличных от многочленов и рациональных функций, давно интересуют математиков и активно изучаются. Одной из первых таких работ можно считать работу Карлемана [1] от 1922 года, в которой установлено, что система $\left\{e^{\lambda_{n} z}\right\}, 0<\lambda_{n}<\infty$, полна в пространстве функций, аналитических в полосе $|\operatorname{Im}(z)|<a$; ширина полосы зависит от плотности последовательности $\left\{\lambda_{n}\right\}$.

В 1938 г. была опубликована работа Гельфонда [2], в которой описаны достаточные признаки полноты систем вида $\left\{f\left(\lambda_{n} z\right)\right\}$, где $f$-целая функция, а $\left\{\lambda_{n}\right\}$-последовательность комплексных чисел с предельной точкой в бесконечности. Эта работа способствовала дальнейшему изучению систем аналитических функций. В 1945 г. публикуется статья Маркушевича [3], в которой для изучения аппроксимативных свойств функциональных последовательностей эффективно использовались методы функционального анализа. На основе этих методов там же был получен известный принцип двойственности Маркушевича.

С 1947 г. системы $\left\{f\left(\lambda_{n} z\right)\right\}$ активно изучаются Леонтьевьм (см. [4]-[8]) и его учениками (Красичков, Напалков, Седлецкий и др.). Интерес к системам $\left\{f\left(\lambda_{n} z\right)\right\}$ связан также и с тем, что такие функции являются простейшими решениями уравнений типа свертки.

В 50-е годы в работах Леонтьева [7], [8] проводится исследование систем $\left\{Y\left(z ; \lambda_{n}\right)\right\}$, где $Y(z ; \lambda)$ - функция, аналитическая по переменной $\lambda \in \mathbb{C}$. В частности, изучаются системы $\left\{Y\left(z ; \lambda_{n}\right)\right\}$ собственных функций (соответствующих собственным числам $\left.\left(\lambda_{n}\right)\right)$ линейных дифференциальных операторов [8].

Красичков [9], [10] провел детальное исследование задач полноты систем $\left\{F\left(z ; \lambda_{n}\right)\right\}$, где $F\left(z ; \lambda_{n}\right)$ - функция двух комплексных переменных, определенная на множестве $P \times G \subset \mathbb{C}^{2}$. Задачам полноты систем вида $\left\{f\left(z+\lambda_{j}\right)\right\}$ посвящены работы Казьмина [11], [12].

В последние годы активно изучают также и многомерные системы вида $\left\{f\left(\lambda_{j}^{(1)} z_{1}, \ldots\right.\right.$, $\left.\left.\lambda_{j}^{(n)} z_{n}\right)\right\}$, в частности, системы многомерных экспонент (см. [13]).

Нетрудно видеть, что указанные вьше и многие другие изучаемые ранее системы представляют собой системы значений (в точках $\left\{\lambda_{j}\right\}$ ) той или иной фиксированной 
вектор-функции (как правило, голоморфной) со значениями в том или ином (функциональном) локально вьпуклом пространстве.

При таком взгляде на рассматриваемые выше системы естественно встает следующая общая задача: провести исследование и установить условия полноты системы значений $\left\{f\left(\lambda_{j}\right)\right\}$ голоморфной вектор-функции $f(\lambda)$ со значениями в произвольном локально выпуклом пространстве. Этой задаче и посвящена настоящая работа. Здесь установлены необходимые и достаточные условия (критерий) полноты системы

$$
\left\{f\left(\lambda_{j}\right)\right\}, \quad\left\{\lambda_{j}\right\} \subset G \subset \mathbb{C}^{n}, \quad n \geqslant 1,
$$

где $f$ - голоморфная (однозначная) в односвязной области $G$ вектор-функция со значениями в пространстве $\Phi$ реше.

В работе разработан общий (операторный) метод исследования задач полноты функциональных систем довольно общей природы. Этот метод, в частности, раскрьвает внутренние связи изучаемых систем и условий их полноты. Классические функциональные системы выступают здесь как простые частные случаи. Полученные общие результаты иллюстрируются разнообразньми примерами.

ПРИмЕчАниЕ. Мы ограничиваемся пространством Фреше, поскольку большинство конкретных функциональных систем, встречающихся на практике, являются системами элементов того или иного пространства Фреше. Однако как нетрудно заметить, многие из проводимых ниже рассуждений и формулировок являются справедливыми и для систем из более общих локально выпуклых пространств.

1. Критерий полноты системы $\left\{f\left(\lambda_{j}\right)\right\}$. Пусть $H$ - полное метризуемое локально выпуклое пространство над полем комплексных чисел (банахово пространство не исключается). И пусть $f(\lambda)$ - слабо голоморфная, однозначная в односвязной области $G \subset \mathbb{C}^{k}, k \geqslant 1$, вектор-функция со значениями в $H$. Не ограничивая общности, полагаем, что $0 \in G$. Известно (см. [14]), что в пространстве Фреше слабая и сильная голоморфность определяют один и тот же класс функций. Такая функция, как известно, в некоторой окрестности нуля представляется степенным рядом:

$$
f(\lambda)=\sum_{(n)} x_{(n)} \lambda^{n}, \quad x_{(n)} \in H, \quad \lambda^{n}=\left(\lambda_{1}^{n_{1}} \cdots \lambda_{k}^{n_{k}}\right),
$$

абсолютно сходящимся по топологии пространства $H$.

Обозначим через $P$ пространство скалярных функций вида

$$
P=\left\{\varphi(\lambda)=l[f(\lambda)]: l \in H^{*}, \lambda \in G\right\},
$$

где $H^{*}$ - пространство, сильно сопряженное с $H$.

Очевидно, что $P$ есть некоторое подпространство пространства скалярных функций, аналитических в области $G$.

ОПРЕДЕЛЕНИЕ 1. Множество $\left\{\lambda_{j}\right\} \subset G$ назьвается множеством единственнос$m u$ пространства $P$, если для любого $\varphi \in P$ из равенств $\varphi\left(\lambda_{j}\right)=0$ при всех $j$ следует, что $\varphi \equiv 0$.

В этих обозначениях справедлива

ТЕорема 1 (критерий). Для того чтобы система $\left\{f\left(\lambda_{j}\right)\right\},\left\{\lambda_{j}\right\} \subset G \subset \mathbb{C}^{n}, n \geqslant 1$, была полна в $H$, необходимо и достаточно выполнения следующих условий:

1) последовательность $\left\{x_{(n)}\right\}$ полна в $H$;

2) $\left\{\lambda_{j}\right\}$-мнохество единственности в $P$. 
ДокАЗАТЕЛЬСтво. Достаточность. Пусть вьполнены условия 1) и 2), а система $\left\{f\left(\lambda_{j}\right)\right\}$ не полна в $H$. Тогда существует функционал $l_{0} \in H^{*}, l_{0} \neq 0$, такой, что $l_{0}\left[f\left(\lambda_{j}\right)\right]=0$ для всех $j$.

Рассмотрим функцию $\varphi_{0}(\lambda)=l_{0}[f(\lambda)]$. Она принадлежит пространству $P$, в некоторой окрестности нуля представляется рядом

$$
\varphi_{0}(\lambda)=\sum_{(n)} l_{0}\left(x_{n}\right) \lambda^{n}, \quad|\lambda|<r_{0}\left(l_{0}\right)
$$

и удовлетворяет условию $\varphi_{0}\left(\lambda_{j}\right)=0$ для всех $j$. Но $\left\{\lambda_{j}\right\}-$ множество единственности в $P$, следовательно, $\varphi_{0} \equiv 0$. Поэтому

$$
l_{0}\left(x_{n}\right)=0 \quad \forall n, \quad l_{0} \neq 0 .
$$

А это означает (согласно критерию Банаха), что система $\left\{x_{n}\right\}$ не полна в $H$. Достаточность доказана.

Необходимость. Пусть система $\left\{f\left(\lambda_{j}\right)\right\}$ полна, а система $\left\{x_{(n)}\right\}$ не полна в $H$. Тогда найдется $l_{1} \in H^{*}, l_{1} \neq 0$, такой, что $l_{1}\left(x_{(n)}\right)=0$ для всех $n$. Следовательно,

$$
l_{1}[f(\lambda)]=\sum_{n=0}^{\infty} l_{1}\left(x_{n}\right) \lambda^{n} \equiv 0, \quad|\lambda|<r_{1} .
$$

Отсюда в силу теоремы единственности для аналитической функции следует, что

$$
\varphi_{1}(\lambda)=l_{1}[f(\lambda)] \equiv 0 \quad \forall \lambda \in G
$$

и потому

$$
\varphi_{1}\left(\lambda_{j}\right)=l_{1}\left[f\left(\lambda_{j}\right)\right] \equiv 0, \quad\left\{\lambda_{j}\right\} \in G .
$$

Но это означает, что система $\left\{f\left(\lambda_{j}\right)\right\}$ не полна в $H$. Полученное противоречие и доказьвает необходимость условия 1$)$.

Пусть теперь система $\left\{f\left(\lambda_{j}\right)\right\}$ полна в $H$, а $\left\{\lambda_{j}\right\}$ не есть множество единственности пространства $P$. Это означает, что в $P$ существует функция

$$
\varphi_{1}(\lambda)=l_{1}[f(\lambda)] \neq 0, \quad l_{1} \in H^{*}, \quad l_{1} \neq 0
$$

такая, что

$$
\varphi_{1}\left(\lambda_{j}\right)=l_{1}\left[f\left(\lambda_{j}\right)\right]=0 \quad \forall j .
$$

Но тогда система $\left\{f\left(\lambda_{j}\right)\right\}$ не полна в $H$, что и доказывает необходимость условия 2 ).

Теорема доказана полностью.

В случае, когда система $\left\{x_{n}\right\}$ не полна в $H$, можно говорить об “относительной полноте" системы $\left\{f\left(\lambda_{j}\right)\right\}$ в пространстве

$$
V=\overline{\mathscr{L}\left\{x_{n}\right\}} \subset H
$$

где $\overline{\mathscr{L}\left\{x_{n}\right\}}$ - замыкание по топологии пространства $H$ линейной оболочки системы $\left\{x_{n}\right\}$.

Для такого случая в качестве следствия теоремы 1 получаем следующее предложение. 
Теорема 2. Пусть в условиях теоремы 1

$$
V=\overline{\mathscr{L}\left\{x_{n}\right\}} \subset H .
$$

Тогда для того чтобы система $\left\{f\left(\lambda_{j}\right)\right\},\left\{\lambda_{j}\right\} \subset G$, была полна в $V$, необходимо и достаточно, чтобы $\left\{\lambda_{j}\right\}$ представляло из себя множество единственности пространства

$$
P=\left\{\varphi(\lambda)=l[f(\lambda)]: l \in V^{*}, \lambda \in G\right\} .
$$

ПримечАнИЕ. Отметим, что проверка условий 1) и 2) теоремы 1 в общем случае представляет трудную задачу, да и пространство $P$ часто имеет довольно сложную структуру. Однако для классических систем $\left\{e^{\lambda_{j} z}\right\},\left\{f\left(\lambda_{j} z\right)\right\}$ и $\left\{f\left(z+\lambda_{j}\right)\right\}$ проверка полноты системы $\left\{x_{n}\right\}$ в рассматриваемых пространствах часто не представляет трудности. А множества $\left\{\lambda_{j}\right\}$ единственности для соответствующего пространства $P$ можно выбирать с определенным "запасом", используя те или иные известные теоремы единственности, что по сушеству и делается в большинстве известных случаев.

Приведем два примера.

ПримеР 1. Пусть $H=s$ - пространство всех числовых последовательностей $x=$ $\left\{x_{n}\right\} \in s$ с локально вьпуклой топологией, задаваемой полунормами

$$
\|x\|_{p}=\max _{k \leqslant p}\left(\left|x_{k}\right|\right), \quad p=1,2, \ldots
$$

Рассмотрим вектор-функцию

$$
f(\lambda)=\sum_{n=0}^{\infty} e_{n} \lambda^{n}
$$

где $e_{n}=(0, \ldots, 0,1,0, \ldots)$ - орты пространства $s$.

Очевидно, что эта вектор-функция целая. Пусть $\left\{\lambda_{j}\right\}$ - любая бесконечная последовательность попарно различных чисел. Тогда система $\left\{f\left(\lambda_{j}\right)\right\}$ полна в $s$. Действительно, система $\left\{e_{n}\right\}$ полна в $s$, a $\left\{\lambda_{j}\right\}$ - множество единственности для соответствующего пространства $P$, т.е. оба условия теоремы 1 вьполнены.

ПримеР 2. Пусть $H=H(G), G \subset \mathbb{C}^{k}, k \geqslant 1,-$ пространство функций аналитических в односвязной области $G$ с топологией равномерной сходимости на компактах. Рассмотрим целую вектор-функцию

$$
f(\lambda)=\sum_{n=0}^{\infty} p_{n}(z) \lambda^{n}, \quad\left\{p_{n}(z)\right\} \subset H(G), \quad f: \mathbb{C} \rightarrow H(G),
$$

(ряд (1.1) сходится абсолютнопо топологии пространства $H(G)$ в каждой точке $\lambda \in \mathbb{C}$ ).

Пусть $f(\lambda)$ имеет в $H(G)$ порядок роста $\rho>0$. Тогда легко проверяется, что последовательность $\left\{\lambda_{j}\right\}$ с показателем сходимости $\tau>\rho$ является множеством единственности для соответствующего пространства $P$, описанного в теореме 1 .

Пусть система $\left\{p_{n}(z)\right\}$ полна в $H(G)$, тогда в силу теоремы 1 система $\left\{f\left(\lambda_{j}\right)\right\}$ полна в $H(G)$. Если же система $\left\{p_{n}(z)\right\}$ не полна в $H(G)$, то система $\left\{f\left(\lambda_{j}\right)\right\}$ полна согласно теореме 2 в пространстве $V=\overline{L\left\{p_{n}(z)\right\}} \subset H(G)$.

Отметим, что в этом примере содержится в качестве частного случая известная классическая теорема Гельфонда [2] о полноте системы $\left\{f\left(\lambda_{j} z\right)\right.$ в пространстве $H(\mathbb{C})$, где $f$ - целая скалярная функция одного переменного, и другие классические случаи.

Примеров (простых и сложных), иллюстрирующих теоремы 1 и 2 , можно приводить довольно много. В п. 3 мы остановимся кратко на некоторых из них, связанных с широко распространенными в практике операторами. Но прежде рассмотрим специальный, но достаточно общий класс векторно-значных функций и применим доказанный выше критерий к такому случаю. 
2. Об одном классе векторно-значных функций. Пусть $A_{1}, \ldots, A_{k}$ - семейство линейных перестановочных друг с другом операторов, действующих в пространстве $H$ с определяющей топологию системой норм $\left\{\|\cdot\|_{p}\right\}, A_{i}(H) \subset H, i=1,2, \ldots$. Пусть $x \in H$ - фиксированньй вектор, на которьй указанные операторы действуют бесконечно много раз.

Для изучения последовательностей $\left\{A_{1}^{n_{1}} \cdots A_{k}^{n_{k}}(x)\right\}$, введем следуюшие характеристики.

ОПРЕДЕЛЕНИЕ 2. Пусть $x \in H$ фиксирован. Число

$$
\begin{gathered}
\varlimsup_{|n| \rightarrow \infty} \frac{\ln \left\|A_{1}^{n_{1}} \cdots A_{k}^{n_{k}}(x)\right\|_{p}}{|n| \ln |n|}=\beta_{p}(x), \\
|n|=n_{1}+n_{2}+\cdots+n_{k}, \quad n_{i}=0,1,2, \ldots, \quad i=1, \ldots, k,
\end{gathered}
$$

назовем операторным р-порядком вектора $x$ относительно семейства операторов $(A)=\left(A_{1}, \ldots, A_{k}\right)$. Точную верхнюю грань

$$
\sup _{(p)}\left\{\beta_{p}(x)\right\}=\beta(x)
$$

назовем операторным порядком вектора $x$ относительно семейства $(A)=$ $\left(A_{1}, \ldots, A_{k}\right)$. Если $A_{1}^{m_{1}} \cdots A_{k}^{m_{k}}(x)=0$ для некоторого набора $\left(m_{1}, \ldots, m_{k}\right)$, то полагаем $\beta_{p}(x)=-\infty$.

ОПРЕДЕЛЕНИЕ 3 . Пусть $\beta_{p}(x) \neq \pm \infty$, число

$$
\alpha_{p}(x)=\varlimsup_{|n| \rightarrow \infty} \frac{\sqrt[|n|]{\left\|A_{1}^{n_{1}} \cdots A_{k}^{n_{k}}(x)\right\|_{p}}}{|n|^{\beta_{p}(x)}}
$$

назовем операторным $p$-типом вектора $x$ относительно семейства $(A)$. Соответственно, число

$$
\alpha(x)=\sup _{(p)}\left\{\alpha_{p}(x)\right\}
$$

назовем типом вектора $x$ относительно семейства $(A)$.

В дальнейшем, если это не ведет к противоречиям, будем говорить кратко о $p$-порядках и $p$-типах вектора.

ПримЕчАниЕ. Как следует из определения, $p$-порядки вектора $\beta_{p}(x)$, могут быть любыми числами: как положительньми, так и отрищательньми, $p$-типы $\alpha_{p}(x)$ всегда неотрицательны.

В случае одного оператора понятие $p$-порядка и $p$-типа вектора введено автором в работах [15], [16].

Пусть, далее

$$
f(z)=\sum_{(n)} a_{(n)} z^{n}, \quad z^{n}=z_{1}^{n_{1}} \cdots z_{k}^{n_{k}} \in \mathbb{C}^{k},
$$

- целая скалярная функция многих комплексных переменных порядка роста (по совокупности переменных) $\rho>0$ и типа $\sigma>0$. Построим функцию

$$
\Phi(t)=f[A(x) t]=\sum_{(n)} a_{(n)} A_{1}^{n_{1}} \cdots A_{k}^{n_{k}}(x) t_{1}^{n_{1}} \cdots t_{k}^{n_{k}},
$$

где $x \in H-$ фиксированньй вектор, $t=\left(t_{1}, \ldots, t_{k}\right) \in \mathbb{C}^{k}, k \geqslant 1$.

Оценивая коэффициенты ряда (2.4) и учитьвая формулы $(2.1)$ и $(2.2)$, стандартным приемом получаем следующие заключения. 
ЛЕмма 1. Если для операторного порядка вектора $x$ выполнена оценка $\beta(x) \leqslant$ $1 / \rho$, а при $\beta(x)=1 / \rho$ его операторный тип $\alpha(x)=0$, то ряд (2.4) сходится абсолютно в $H$ в каждой точке $t \in \mathbb{C}^{k}$ и представляет иелую вектор-функиию $\Phi(t)$ со значениями в $H$.

ЛЕМма 2. Пусть существует предел

$$
\lim _{|n| \rightarrow \infty}|n|^{1 / \rho} \sqrt[|n|]{\left|a_{(n)}\right|}=(\sigma e \rho)^{1 / \rho}, \quad a_{(n)} \neq 0 \quad \forall n, \quad|n|=n_{1}+\cdots+n_{k} .
$$

Тогда в условиях леммы 1 иелая вектор-функиия $\Phi(t)=f[A(x) t]$ имеет следуюшие р-порядки:

$$
R_{p}(\Phi)=\frac{\rho}{1-\rho \beta_{p}(x)}, \quad \beta_{p}(x) \leqslant \frac{1}{\rho} \quad \forall p .
$$

Если при этом $\beta_{p}(x)<1 / \rho$, mо ее $p$-типь

$$
\omega_{p}(\Phi)=\frac{1}{e R_{p}(\Phi)}(\sigma e \rho)^{R_{p}(\Phi) / \rho}\left[\alpha_{p}(x)\right]^{R_{p}(\Phi)},
$$

әде $\alpha_{p}(x)$ - операторный $p$-тип вектора $x$.

Вектор-функция (2.4) содержит в себе в качестве частных случаев большой набор конкретных функций (порождающих функций), которые используются для построения многих известных классических функциональных систем. К ним относятся системы экспонент (порождающая функция $\left\{e^{\lambda z}\right\}$ ), системы обобщенных экспонент (порождающая функция $\{f(\lambda z)\})$, системы сдвигов и их обобщений и др. Эти системы порождаются конкретными операторами, действующими в конкретных функциональных пространствах. Ниже мы рассмотрим такие примеры. Но вначале остановимся на общих системах, порождаемых функцией (2.4), и установим общий критерий полноты системы вида $\left\{\Phi\left(\lambda_{j}\right)=f\left[A(x) \lambda_{j}\right]\right\}$.

Условия полноты указанных систем тесно связаны с понятием циклического вектора.

ОПРЕДЕЛЕНИЕ 4. Вектор $x \in H$ называется ииклическим вектором семейства операторов $(A)=\left(A_{1}, \ldots, A_{k}\right)$, если в пространстве полна система $\left\{A_{1}^{n_{1}} \cdots A_{k}^{n_{k}}(x)\right\}$, $n_{i}=0,1, \ldots, i=1,2, \ldots, k$.

Циклические векторы имеют давнюю историю. Они широко используются в алгебре (циклическая групп, циклическая алгебра). Хорошо изучены циклические векторы в конечномерных пространствах. Часто появляются циклические векторы в теории гильбертовых пространств. В бесконечномерных пространствах они используются в задачах аппроксимации, при исследовании инвариантных подпространств и т.д. Известны исследования циклических векторов оператора умножения $A(F)=z F$, оператора дифференцирования $A(F)=d F / d z$ и др. Циклические векторы оператора дифференцирования в пространствах аналитических функций изучались в работах Маркушевича [3], Казьмина [11], Попова [17]. В работе автора [18] установлены необходимые и достаточные условия цикличности функции относительно оператора дифференцирования и оператора обобщенного дифференцирования Гельфонда-Леонтьева.

В настоящей работе циклические векторы используются в задачах о полноте функциональных систем.

Одним из частных случаев теоремы 1 (случай функции (2.4)), в котором участвуют циклические векторы, является следующее 
ПРЕДЛОЖЕНИЕ 1. Пусть $(A)=\left(A_{1}, \ldots, A_{k}\right)$ - семейство линейных перестановочных операторов, действуюших в $H, u f-$ иелая скалярная функция порядка роста $\rho>0$, типа $\sigma<\infty$. Пусть, далее вектор $x \in H$ имеет операторный порядок $\beta(x) \leqslant 1 / \rho$, а при $\beta(x)=1 / \rho$ его операторный тип $\alpha(x)=0$. Тогда для того чтобы система $\left\{f\left[A(x) \lambda_{j}\right]\right\}$ была полна в $H$, необходимо и достаточно выполнения следуюших условий:

1) $x$ - ииклический вектор семейства операторов $(A)$ в $H$;

2) $\left\{\lambda_{j}\right\}$-множество единственности пространства

$$
P=\left\{\varphi(t)=l\{f[A(x) t]\}: l \in H^{*}\right\},
$$

әде $H^{*}$ - пространство, сильно сопряженное с $H$.

Отметим, что при определении множества $\left\{\lambda_{j}\right\}$ единственности пространства $P$ удобно пользоваться следующей известной оценкой:

$$
\forall \varphi \in P \quad|\varphi(t)|=|l\{f[A(x) t]\}| \leqslant C(l)\|f[A(x) t]\|_{p_{1}}, \quad t \in \mathbb{C}^{k}, \quad k \geqslant 1,
$$

где $C$-постоянная, зависящая от $l \in H^{*}, p_{1}$ - номер нормы, по которой ограничен функционал $l$. Оценка (2.7) показывает, что множество $\left\{\lambda_{j}\right\}$ можно определять с помощью характеристик роста функции $\Phi(t)=f[A(x) t]$.

ПРИмЕчАНИЕ. Предложение 1 представляет критерий полноты систем (вообе говоря, многомерных), которые порождаются функцией вида (2.4). Такая функция порождает, в частности, и следующие распространенные в практике системы: $\left\{f\left(\lambda_{n} z_{1}, \mu_{k} z_{2}\right)\right\},\left\{F\left(z_{1}+\lambda_{n}, z_{2}+\mu_{k}\right)\right\}, n, k=1,2, \ldots$, где $f$ и $F$ - целые функции. Легко показать, что первая система образуется с помощью семейства операторов умножения: $A_{i}(F)=z_{i} F, i=1,2$; вторая - семейством операторов частного дифференцирования: $A_{i}(F)=\partial F / \partial z_{i}, i=1,2$. Обе системы безусловно представляют определенный интерес. В силу предложения 1 необходимьм условием полноты таких систем является условие цикличности некоторого вектора относительно семейства указанных операторов. Но функции циклические относительно семейства операторов в настоящее время слабо изучены. И, по-видимому, исследование многомерных систем операторньм методом - дело будущего.

В данной же работе мы иллюстрируем возможности представленного операторного метода, ограничиваясь одномерным случаем, используя известные результаты о циклических функциях операторов умножения и дифференцирования. Переход к многомерному случаю хотя и просматривается, но он потребует отдельного исследования.

Итак, пусть

$$
f(z)=\sum_{n=0}^{\infty} a_{n} z^{n}, \quad a_{n} \neq 0, \quad z \in \mathbb{C},
$$

- целая скалярная функция порядка $\rho \geqslant 1$ и типа $\sigma<\infty$, удовлетворяющая условию

$$
\lim _{n \rightarrow \infty} n^{1 / \rho} \sqrt[n]{\left|a_{n}\right|}=(e \rho \sigma)^{1 / \rho}
$$

и пусть

$$
\Phi(t)=f[A(x) t]=\sum_{n=0}^{\infty} a_{n} A^{n}(x) t^{n}, \quad x \in H .
$$

В этих обозначениях в качестве следствия предложения 1 получаем следующее заключение. 
Теорема 3. Пусть $A$ - линейный непрерывный оператор, действующий в $H$, $x \in H$ - ииклический вектор этого оператора - имеет операторный порядок $\beta(x)<1 / \rho$. Тогда если показатель сходимости последовательности $\left\{\lambda_{j}\right\}$ удовлетворяет условию

$$
\tau>\frac{\rho}{1-\rho \beta(x)}
$$

то система $\left\{f\left[A(x) \lambda_{j}\right]\right\}$ полна в $H$.

ДокАЗАТЕЛЬСТво. Для доказательства теоремы в силу предложения 1 достаточно убедиться, что множество $\left\{\lambda_{j}\right\}$ с указанным в теореме условием является множеством единственности пространства $P$. Но это действительно так, поскольку функция $\Phi(t)=f[A(x) t]$ в условиях теоремы целая (лемма 1$)$ и ее порядок роста

$$
R(\Phi)=\frac{\rho}{1-\rho \beta(x)}
$$

(лемма 2). Поэтому, в силу оценки (2.7) и условия $\rho>R(\Phi)$ последовательность $\left\{\lambda_{j}\right\}-$ множество единственности пространства $P$. Теорема доказана.

Отметим, что частным случаем функции (2.9) является вектор-функция

$$
\Phi(t)=e^{t A}(x)=\sum_{n=0}^{\infty} \frac{A^{n}(x)}{n !} t^{n}, \quad x \in H
$$

(случай $\left.f(z)=e^{z}\right)$.

Такая функция используется в теории дифференциально-операторных уравнений и часто назьвается операторной әкспонентой. В определенных условиях она представляет решение следуюшей задачи Коши:

$$
\frac{d u}{d t}=A(u), \quad u(0)=x \in H .
$$

Теорема 3 содержит в себе условия полноты систем $\left\{e^{\lambda_{j} A}(x)\right\}$. В силу предложения 1 необходимьм условием полноты такой системы является цикличность вектора $x \in H$ относительно оператора $A$. В частности, если $A$-оператор умножения $A(F)=z(F)$, действующий в некотором фиксированном пространстве, то, полагая $F(z) \equiv 1$, получаем функцию $\Phi(t)=e^{t z}$, порождающую классические системы экспонент. Задачам полноты систем экспонент посвящено большое число исследований. Изучались они в различных функциональных пространствах, применялись разнообразные методы. Представленньй общий операторньй метод открьвает возможности единообразного исследования большого класса систем вида $\left\{e^{\lambda_{j} A}(x)\right\}$, обобщающих системы экспонент. При этом проявляется и внутренняя сущность рассматриваемых задач. Так, например, в силу предложения 1 полнота систем $\left\{e^{\lambda_{j} z}\right\}$ возможна только в тех пространствах, в которых функция $F \equiv 1$ циклическая для оператора умножения (необходимое условие полноты).

Рассмотрим еще один интересный вариант порождающих функций и соответствующих им систем.

Как известно, операторные порядки вектора могут быть отрицательными. Такие ситуации на практике часто встречаются. В этом случае нетрудно заметить, что функция

$$
\Phi(t)=\sum_{n=0}^{\infty} A^{n}(x) t^{n}
$$


целая (ряд сходится абсолютно в $H$ в каждой точке $t \in \mathbb{C}$ ). Характеристики роста функции (2.10) легко посчитать. Они равны соответственно

$$
R_{p}(\Phi)=-\frac{1}{\beta_{p}(x)}, \quad \omega_{p}(\Phi)=-\frac{\beta_{p}(x)}{e}\left[\alpha_{p}(x)\right]^{R_{p}(\Phi)} \quad \forall p,
$$

где $\beta_{p}(x)<0$ - операторные $p$-порядки, а $\alpha_{p}(x)$ - операторные $p$-типы вектора $x$.

Для такого случая из предложения 1 и теоремы 3 следует

Teорема 4. Пусть оператор А действует в пространстве $H$ u $x \in H-u$ иллический вектор этого оператора, имеющий отрицательные $p$-порядки $\beta_{p}(x)$. Тогда

1) ряд (2.10) представляет целую вектор-функиию $\Phi(t): \mathbb{C} \rightarrow H$, характеристики роста которой (р-порядки и р-типь) вычисляются по формулам (2.11);

2) если последовательность $\left\{\lambda_{j}\right\}$ имеет показатель сходимости $\tau>-1 / \beta_{p}(x)$ при всех р, то система $\left\{\Phi\left(\lambda_{j}\right)\right\}$ полна в $H$.

ДоКАЗАТЕЛЬСТВО этой теоремы сводится к вычислению характеристик роста функции $\Phi(t)$ по стандартным формулам. Затем применяется (в условиях теоремы) предложение 1 и теорема 3 к рассматриваемой системе.

Не безынтересно отметить, что функция (2.10) является решением операторного уравнения

$$
u-t A(u)=x, \quad x \in H
$$

Формально функцию (2.10) можно записать в следующей операторной форме:

$$
\Phi(t)=(E-t A)^{-1}(x)
$$

Теорему 4 можно рассматривать и как теорему о полноте системы решений

$$
\Phi\left(\lambda_{j}\right)=\left(E-\lambda_{j} A\right)^{-1}(x),
$$

соответствуюших уравнению (2.12) и параметрам $\left\{\lambda_{j}\right\}$. Здесь также необходимым условием полноты является цикличность вектора $x$ относительно оператора $A$.

Теоремы 3, 4 и предложение 1 представляют закономерности общего характера, справедливые для операторов, действуюших в произвольных локально вьпуклых пространствах. В следующем пункте они иллюстрируются на конкретных функциональных пространствах с помощью конкретных операторов.

ПРИмЕчАниЕ. Отметим, что в теоремах 3 и 4 для определения множества $\left\{\lambda_{j}\right\}$ используется одна из известньх теорем единственности теории целых функций, связывающая показатель сходимости $\tau$ с порядком роста функции. Разумеется, можно пользоваться и другими теоремами единственности. И каждая из них будет поставлять определенное заключение о полноте рассматриваемых систем.

3. Примеры. В этом пункте демонстрируется предложенньй выше операторный метод на некоторых распространенных на практике функциональных последовательностях. При этом используются операторы умножения и оператор дифференцирования, действующие в некоторых пространствах целых функций. 
ПримеР 3. Полнота системы $\left\{f\left(\lambda_{j} z\right)\right\}$ в пространстве $\left[\rho_{1}, \sigma_{1}\right]$.

Пусть $A$-оператор умножения: $A(F)=z F(z)$, действуюший в пространстве $\left[\rho_{1} ; \sigma_{1}\right]$ целых функций порядка $\leqslant \rho_{1}$, а при $\rho=\rho_{1}$ типа $\leqslant \sigma_{1}<\infty$. Топологию в $\left[\rho_{1} ; \sigma_{1}\right]$ определим системой норм:

$$
\|F\|_{\varepsilon}=\sup _{r>0}\left\{\max _{|z| \leqslant r}\left[|F(z)| e^{-\left(\sigma_{1}+\varepsilon\right) r_{1}^{\rho}}\right]\right\} \quad \forall \varepsilon>0, \quad \forall F \in\left[\rho_{1}, \sigma_{1}\right] .
$$

С этой топологией $\left[\rho_{1} ; \sigma_{1}\right]$ - полное метрическое пространство (пространство Фреше) и $A$ - в нем линейный и непрерьвньй оператор. Известно (см. [19], [20]), что его порядок $\beta(A)=1 / \rho_{1}$ и тип $\alpha(A)=\infty$.

Пусть $f$ - целая скалярная функция порядка $\rho<\rho_{1}$, типа $\sigma<\infty$, удовлетворяющая условию (2.8). Построим по правилу (2.4) вектор-функцию

$$
\Phi(t)=f[A(F) t]=\sum a_{n} A^{n}(F) t^{n}=F(z) f(z t), \quad F \in\left[\rho_{1}, \sigma_{1}\right] .
$$

Легко проверяется, что ряд (3.1) сходится абсолютно в пространстве $\left[\rho_{1}, \sigma_{1}\right]$ в каждой точке $t \in \mathbb{C}$ и представляет целую вектор-функцию $\Phi(t): \mathbb{C} \rightarrow\left[\rho_{1}, \sigma_{1}\right]$. В дальнейшем мы ограничиваемся случаем $F \equiv 1$, т.е. изучаем функцию $\Phi(t)=f(z t)$, которая порождает системы $\left\{f\left(\lambda_{j} z\right)\right\}$. Непосредственным подсчетом находим

$$
\left\|A^{n}(1)\right\|_{\varepsilon}=\left\|z^{n}\right\|_{\varepsilon}=\left[\frac{n}{e\left(\sigma_{1}+\varepsilon\right) \rho_{1}}\right]^{n / \rho_{1}}, \quad \varepsilon>0, \quad n=0,1, \ldots
$$

Эти равенства показывают, что операторные порядки и типы вектора $F \equiv 1$ относительно оператора $A$ соответственно равны

$$
\begin{gathered}
\beta_{\varepsilon}(1)=\frac{1}{\rho_{1}}=\beta(1), \quad \alpha_{\varepsilon}(1)=\left(\frac{1}{e\left(\sigma_{1}+\varepsilon\right) \rho_{1}}\right)^{1 / \rho_{1}} \\
\alpha(1)=\left(\frac{1}{e \rho_{1} \sigma_{1}}\right)^{1 / \rho_{1}}, \quad \varepsilon>0 .
\end{gathered}
$$

Пользуясь этими равенствами по формулам (2.5) и (2.6) находим характеристики роста функции $\Phi(t)=f(z t)$ :

$$
\begin{gathered}
R_{\varepsilon}(\Phi)=\frac{\rho \rho_{1}}{\rho_{1}-\rho}=R(\Phi) \quad \forall \varepsilon>0 . \\
\omega_{\varepsilon}(\Phi)=\left(\frac{\rho_{1}-\rho}{\rho \rho_{1}}\right)(\sigma \rho)^{\rho_{1} /\left(\rho_{1}-\rho\right)}\left[\frac{1}{\left(\sigma_{1}+\varepsilon\right) \rho_{1}}\right]^{\rho /\left(\rho_{1}-\rho\right)} \quad \forall \varepsilon>0 .
\end{gathered}
$$

ПРИмечАниЕ. Отметим, что операторные типы векторов $F \in\left[\rho_{1}, \sigma_{1}\right]$, как показано Мишиньм [19], [20], могут быть как угодно большими.

Пользуясь операторньм методом в этом конкретном случае в качестве одного из следствий теоремы 3 получаем следующее утверждение. 
Теорема 5. Пусть $f$ - целая скалярная функция порядка роста $\rho>0$, типа $\sigma<\infty$, удовлетворяющая условию (2.8). И пусть $\tau$ - показатель сходимости последовательности $\left\{\lambda_{j}\right\}$. Тогда

1) вектор-функиия

$$
\Phi(t)=f(z t): \mathbb{C} \rightarrow[\rho, \sigma], \quad \rho<\rho_{1},
$$

целая; ее характеристики роста вычисляются по формулам (3.2) и (3.3);

2) если $\tau>\rho \rho_{1} /\left(\rho_{1}-\rho\right)$, то система $\left\{f\left(\lambda_{j} z\right)\right\}$ полна в пространстве $\left[\rho_{1} ; \sigma_{1}\right]$, $\rho_{1}>\rho$

3) если $\tau<\rho \rho_{1} /\left(\rho_{1}-\rho\right)$, то существует линейный непрерывный оператор $B$, определенный в пространстве $\left[\rho_{1}, \sigma_{1}\right]$, ядро которого $\operatorname{Ker}(B)=\overline{\mathscr{L}\left\{f\left(\lambda_{j} z\right)\right\}}$, где $\overline{\mathscr{L}\left\{f\left(\lambda_{j} z\right)\right\}}$ - замкнутая в $\left[\rho_{1}, \sigma_{1}\right]$ линейная оболочка системы $\left\{f\left(\lambda_{j} z\right)\right\}$; в этом случае система $\left\{f\left(\lambda_{j} z\right)\right\}$ в $\left[\rho_{1}, \sigma_{1}\right]$ не полна, но если $\rho<\tau<$ $\rho \rho_{1} /\left(\rho_{1}-\rho\right)$, то она полна в пространстве $H(\mathbb{C})$ всех иельх функиий $c$ топологией равномерной сходимости на компактах.

ДокАЗАТЕЛьство. Первое утверждение получаем непосредственным подсчетом, используя операторные порядки (3.2) функции $F \equiv 1$.

Второе утверждение есть следствие теоремы 3 , если учесть, что $\tau>R(\Phi)$ и воспользоваться оценкой (2.7).

Для получения третьего утверждения пользуемся результатом Леонтьева (см. [7, c. 117, лемма 1.6.3]) в силу которого, в условиях п. 3), существует целая функция $\varphi(t)=$ $\sum_{n=0}^{\infty} c_{n} t^{n}$ порядка $\rho_{2}=\tau<\rho \rho_{1} /\left(\rho_{2}-\rho\right)$ с нулями $\left\{\lambda_{j}\right\}$, определяющая на $\left[\rho_{1}, \sigma_{1}\right]$ линейньй непрерьвный оператор

$$
B(F) \equiv \varphi(D) F \equiv \sum_{n=0}^{\infty} c_{n} D^{n} F,
$$

где $D$ - оператор обобщенного дифференцирования Гельфонда-Леонтьева с условием $D f\left(\lambda_{j} z\right)=\lambda_{j} f\left(\lambda_{j} z\right)$. Именно у этого оператора ядро равно $\overline{\mathscr{L}\left\{f\left(\lambda_{j} z\right)\right\}}$. Отсюда следует, что система $\left\{f\left(\lambda_{j} z\right)\right\}$ в $\left[\rho_{1}, \sigma_{1}\right]$ не полна. Однако, если $\rho<\tau<\rho \rho_{1} /\left(\rho_{1}-\rho\right)$, то по теореме Гельфонда [2] такая система полна в пространстве $H(\mathbb{C})$.

Теорема доказана.

Приведем еще одно следствие теоремы 3 , в котором для определения множества $\left\{\lambda_{j}\right\}$, привлекается теорема единственности, связьвающая максимальную плотность последовательности $\left\{\lambda_{j}\right\}$ с порядком и типом целой функции. Именно, справедлива

ТЕОРема 6. Пусть в условиях теоремы 5 выполняется условие

$$
\varlimsup_{n \rightarrow \infty} \frac{n}{\left|\lambda_{n}\right|^{\tau}}>e(\sigma \rho)^{\tau / \rho}\left(\rho_{1} \sigma_{1}\right)^{-\tau / \rho_{1}}, \quad \tau=\frac{\rho \rho_{1}}{\rho_{1}-\rho}, \quad \sigma_{1}>0 .
$$

Тогда система $\left\{f\left(\lambda_{j} z\right)\right\}$ полна в $\left[\rho_{1}, \sigma_{1}\right]$.

ПримЕчАниЕ. Системы $\left\{f\left(\lambda_{j} z\right)\right\}$ активно изучались во второй половине прошлого века. Изучаются они и в настоящее время. Основные результаты этого направления полно представлены в монографии Леонтьева [7]. Содержание теорем 5 и 6 тесно соприкасается, а иногда и пересекается с аналогичными результатами Леонтьева, полученньми им ранее другими методами (не используя методы функционального анализа). Отметим лишь, что утверждение 1 теоремы 5 и теорема 6 в доступной литературе не обнаружены. Вероятно, они отсутствуют. Утверждения 2) и 3) теоремы 5 известны. Они устанавливались ранее другими методами. Информация об этом имеется в монографиии [7].

Рассмотрим другой пример, иллюстрирующий предложение 1. 
ПримеР 4. Пусть $H$ - некоторое полное топологическое пространство цельх функций. В этом пространстве рассмотрим оператор $A=d / d z$. Пусть, далее, $f$ - фиксированная скалярная целая функция порядка $\rho>0$, типа $\sigma<\infty$, удовлетворяющая условию (2.8). Если операторный порядок функции $F \in H$ удовлетворяет условию $\beta(F)<1 / \rho$, то в силу лемм 1 и 2 формула (2.9) представляет целую векторно-значную функцию

$$
\Phi(t)=f[A(F) t]=\sum_{n=0}^{\infty} a_{n} F^{(n)}(z) t^{n}
$$

со значениями в пространстве $H$. Характеристики роста функции (3.4) определяются по формулам (2.5) и (2.6).

Функции вида (3.4) представляют обобщение функции сдвига $\Phi(t)=F(t+z)$, которая, очевидно, следует из (3.4) при $f(z)=e^{z}$. Поэтому такие функции назьвают обобщенным сдвигом, порохденным функиией $f$.

Сформулируем следующее следствие предложения 1.

СлЕдСтвиЕ 1. Пусть $f-$ иелая функиия порядка роста $\rho>0$, muпа $\sigma<\infty$ удовлетворяет условию (2.8). И пусть операторный порядок (относительно оператора дифферениирования) функиии $F \in H$ удовлетворяет условию $\beta(F)<1 / \rho$. Для того чтобы система $\left\{\Phi\left(\lambda_{j}\right)\right\}$ была полна в $H$, необходимо и достаточно, чтобы выполнялись следующие условия:

1) $F-$ ииклическая функиия оператора $d / d z$ в $H$;

2) $\left\{\lambda_{j}\right\}$-мнохество единственности следующего пространства щельх функu,uั̆

$$
P=\left\{\varphi(t)=l\{f[A(F) t]\}: \forall l \in H^{*}\right\}
$$

Отметим, что следствие 1 содержит в себе в качестве частного случая необходимое и достаточное условие полноты системы сдвигов $\left\{F\left(z+\lambda_{j}\right)\right\}$ (случай $\left.f(z)=e^{z}\right)$. Такие системы различными методами изучались в работах Маркушевича [3], Казьмина [11] и др. Следствие 1 показывает, что необходимым условием полноты системы $\left\{F\left(z+\lambda_{j}\right)\right\}$ является цикличность функции $F$. относительно оператора дифференцирования. В работе автора [18], посвященной циклическим функциям оператора дифференцирования, доказана

ТЕоремА В. Каждая иелая функиия әкспоненииального типа, не являющаяся конечной линейной комбинацией корневых функций оператора $d / d z$, представляет собой ииклическую функиию оператора $d / d z$ в пространстве $H(G)$, где $G \subset \mathbb{C}-$ односвязная область.

По этой теореме функции

$$
\cos \sqrt{z}, \quad \frac{\sin z}{z}, \quad \frac{e^{z}-1}{z}
$$

являются циклическими для оператора $A=d / d z$. Поэтому из следствия 1 , с учетом того факта, что вектор-функция $\Phi(t)=F(z+t)$ имеет порядок роста, равный порядку роста целой функции $F(z)$, вытекают следующие заключения:

1) система $\left\{\cos \sqrt{z+\lambda_{j}}\right\}$ полна в $H(G)$, если показатель сходимости последовательности $\left\{\lambda_{j}\right\} \tau>1 / 2$;

2) системы

$$
\left\{\frac{\sin \left(z+\lambda_{j}\right)}{z+\lambda_{j}}\right\}, \quad\left\{\frac{e^{\left(z+\lambda_{j}\right)}-1}{z+\lambda_{j}}\right\}
$$


полны в $H(G)$, если $\tau>1$.

В заключение приведем пример, иллюстрирующий теорему 4.

ПРИмеР 5. Пусть $H=H(\mathbb{C})$ - пространство всех целых функций, и пусть

$$
\Phi(t)=\sum_{n=0}^{\infty}(\cos \sqrt{z})^{(n)} t^{n}
$$

Операторный порядок $\beta(\varphi)$ целой функции $\varphi(z)=\cos \sqrt{z}$ относительно оператора дифоференцирования в пространстве $H(\mathbb{C})$ легко считается, и он равен $\beta(\varphi)=-1$. Следовательно ряд (3.5) сходится абсолютно в $H(\mathbb{C})$ в каждой точке $t \in \mathbb{C}$ и представляет целую вектор-функцию $\Phi(t): \mathbb{C} \rightarrow H(\mathbb{C})$. Характеристики роста функции $\Phi(t)$ выгисляются по формулам (2.10) и они равны

$$
R_{p}(\Phi)=R(\Phi)=-\frac{1}{\beta(\varphi)}=1, \quad \omega_{p}(\Phi)=\frac{1}{e}\left[\alpha_{p}(\varphi)\right], \quad \forall p
$$

где $\alpha_{p}(\varphi)$ - операторньй $p$-тип функции $\varphi$. Легко находим $\alpha_{p}(\varphi)=e / 4$ при всех $p$. Следовательно $\omega(\Phi)=1 / 4$. Функция $\cos \sqrt{z}$, как уже отмечалось, циклическая.

В качестве следствия теоремы 4 получаем следующее

УТВЕРЖДЕНИЕ. Если последовательность $\left\{\lambda_{j}\right\}$ удовлетворяет условию

$$
\varlimsup_{n \rightarrow \infty} \frac{n}{\left|\lambda_{n}\right|}>\frac{e}{4}
$$

то система $\left\{\Phi\left(\lambda_{j}\right)\right\}$ полна в $H(\mathbb{C})$.

ПРИмЕчАнИЕ. Отметим, что функция $\Phi\left(\lambda_{j}\right)$ является решением уравнения

$$
u-\lambda_{j} u^{\prime}(z)=\cos \sqrt{z} \text {. }
$$

Поэтому последнее утверждение говорит о полноте системы решений уравнения (3.6), соответствующих параметрам $\left\{\lambda_{j}\right\}$, в пространстве $H(\mathbb{C})$.

Не безьнтересно отметить, что решением уравнения

$$
u-\lambda \frac{d u}{d z}=\frac{\sin z}{z}
$$

является вектор-функция

$$
u(\lambda)=\sum_{n=0}^{\infty}\left(\frac{\sin z}{z}\right)^{(n)} \lambda^{n}, \quad|\lambda|<1
$$

Функция $(\sin z) / z$ циклическая относительно оператора дифференцирования, поэтому в силу следствия 1 система $\left\{u\left(\lambda_{j}\right)\right\}$ полна в пространстве $H(\mathbb{C})$, если $\left\{\lambda_{j}\right\}$ - множество единственности для пространства функций, аналитических в круге $|\lambda|<1$. 


\section{СПИСОК ЦИТИРОВАННОЙ ЛИТЕРАТУРЫ}

[1] Carlemann T. Über die Approximation analytischer Function durch lineare Aggregate von vorgegebenen Potenzen // Arkiv für Math. Astr. och Fysic. 1922. V. 17. № 9. P. 1-30.

[2] Гельфонд А. О. О полных системах аналитических функций // Матем. сб. 1938. Т. 4 (46). C. $149-156$.

[3] Маркушевич А. И. О базисе в пространстве аналитических функций // Матем. сб. 1945. T. 17 (59). C. 211-252.

[4] Леонтьев А.Ф. О полноте одной системы аналитических функций // Матем. сб. 1952. T. 31 (73). C. $318-413$.

[5] Леонтьев А.Ф. Ряды полиномов Дирихле и их обобщения // Тр. МИАН. 1951. Т. 39. C. $3-212$.

[6] Леонтьев А. Ф. Ряды экспонент. М.: Наука, 1976.

[7] Леонтьев А. Ф. Обобщение рядов экспонент. М.: Наука, 1981.

[8] Леонтьев А.Ф. О последовательностях линейных агрегатов, образованных из решений дифференциальных уравнений // Изв. АН СССР. Сер. матем. 1958. Т. 22. № 2. С. 201-242.

[9] Красичков И.Ф. О полноте функций вида $\frac{\partial^{n_{k}}}{\partial h^{n_{k}}} F\left(z ; h_{k}\right) / /$ Матем. сб. 1962. Т. 56 (98). C. $147-172$.

[10] Красичков И. Ф. Полнотав пространствах комплекснозначных функций, описьваемых поведением модуля // Матем. сб. 1965. Т. 68 (110). № 1. С. 26-57.

[11] Казьмин Ю. А. О полноте систем функций вида $\left\{f\left(z+\alpha_{n}\right)\right\}$ и $\left\{f^{(n)}(z)\right\} / /$ УМН. 1957. T. 12. № 2. C. 151-154.

[12] Казьмин Ю. А. О полноте одной системы аналитических функций I // Вестн. МГУ. Сер. матем. 1956. № 5. С. 3-12.

[13] Громов В.П. О последовательностях линейных агрегатов функций нескольких комплексных переменньх // Докл. АН СССР. 1968. Т. 180. № 4. С. 778-782.

[14] Рудин У. Функциональный анализ. М.: ИЛ, 1975.

[15] Громов В. П. Порядок и тип линейного оператора и разложение в рядпо собственным функциям // Докл. АН СССР. 1986. Т. 228. №1. С. 27-31.

[16] Громов В.П. Порядок и тип линейного оператора и целые векторнозначные функции // Ученые записки Орловского гос. университета. Орел: ОГУ, 1999. С. 6-23.

[17] Попов А. Ю. Об аппроксимативных свойствах некоторых типов последовательностей целых функций // Докл. РАН. 1999. Т. 106. №1. С. 18-20.

[18] Громов В. П. К вопросу о циклических элементах оператора дифференцирования в пространствах аналитических функций // Матем. заметки. 1983. Т. 33. № 4. С. 529-538.

[19] Мишин С. Н. О порядке и типе оператора // Докл. РАН. 2001. Т. 381. № 3. С. 309-312.

[20] Мишин С. Н. Порядок и тип оператора и последовательности операторов, действуюших в локально выпуклых пространствах // Ученые записки Орловского гос. университета. Орел: ОГУ, 2002. № 3. С. 47-99.

[21] Громов В. П. О полноте систем производных аналитических функций // Изв. АН СССР. Сер. матем. 1961. Т. 25. № 4. С. 548-556. 